\title{
A CT reconstruction approach from sparse projection with adaptive-weighted diagonal total-variation in biomedical application
}

\author{
Luzhen Deng ${ }^{\mathrm{a}}$, Deling $\mathrm{Mi}^{\mathrm{a}}{ }^{,}$, Peng He ${ }^{\mathrm{a}}$, Peng Feng ${ }^{\mathrm{a}}$, Pengwei Yu ${ }^{\mathrm{a}}$, Mianyi Chen ${ }^{\mathrm{a}}$, Zhichao Li ${ }^{\mathrm{b}}$, \\ Jian Wang ${ }^{\text {b** }}$ and Biao Wei ${ }^{\mathrm{a}}$ \\ ${ }^{a}$ Key Laboratory of Opto-Electronics Technology \& System, Chongqing University, Ministry of \\ Education, Chongqing 400044, China \\ ${ }^{b}$ The Radiology Department, Affiliated Southwest Hospital, Third Military Medical University, \\ Chongqing 400038, China
}

\begin{abstract}
For lack of directivity in Total Variation (TV) which only uses x-coordinate and y-coordinate gradient transform as its sparse representation approach during the iteration process, this paper brought in Adaptive-weighted Diagonal Total Variation (AwDTV) that uses the diagonal direction gradient to constraint reconstructed image and adds associated weights which are expressed as an exponential function and can be adaptively adjusted by the local image-intensity diagonal gradient for the purpose of preserving the edge details, then using the steepest descent method to solve the optimization problem. Finally, we did two sets of numerical simulation and the results show that the proposed algorithm can reconstruct high-quality CT images from few-views projection, which has lower Root Mean Square Error (RMSE) and higher Universal Quality Index (UQI) than Algebraic Reconstruction Technique (ART) and TV-based reconstruction method.
\end{abstract}

Keywords: Computed tomography (CT), sparse projection, adaptive-weighted diagonal total variation (AwDTV), compressive sensing (CS)

\section{Introduction}

How to reconstruct high-quality Computed Tomography (CT) images from few-views or sparse-views data is a hot research spot in medical CT field [1-4]. The essence of sparse-views reconstruction is trying to find the optimization solution of underdetermined equation. But traditional CT reconstruction algorithms such as Filtered Backprojection (FBP) [5], Algebraic Reconstruction Technique (ART) [6] and Simultaneous Algebraic Reconstruction Techniques (SART) [7] can't reconstruct high quality $\mathrm{CT}$ images with the sparse sampling or limited projection data because of

\footnotetext{
* Address for correspondence: Deling Mi, Key Laboratory of Opto-Electronics Technology \& System, Chongqing University, Ministry of Education, Chongqing 400044, China. Tel.: +02365111163; Fax: +02365111163; E-mail: mideling@163.com.

Jian Wang, The Radiology Department, Affiliated Southwest Hospital, Third Military Medical University, Chongqing 400038, China. Tel.: +02368754000; Fax:+02368754000; E-mail: wangjian811@gmail.com.
} 
their limits in astringency, noise immunity and robustness. Compressive Sensing (CS) theory [8], which points out CT images can be reconstructed from sparse projection date if it can be sparsely depicted in an appropriate domain and the quality of reconstructed CT images depends on the way by which CT images are sparsely represented, makes it possible to get CT images with high quality from sparse projection data.

Total-Variation-based algorithm (TV) $[9,10]$ which is proposed by E.Y. Sidky and X.C. Pan in 2007 is one of the most popular algorithms inspired by CS [9-17]. TV uses the x-coordinate and $\mathrm{y}$-coordinate gradient operator as the sparse representation approach during the iteration process. It can't take full advantage of directional information of edges and textures in CT images. It means that $\mathrm{x}$-coordinate and $\mathrm{y}$-coordinate gradient operator is not the best way to represent images sparsely and can be improved.

Based on this, we propose an AwDTV based CT reconstruction algorithm aiming at exploring the sparse capability of AwDTV to reconstruct better CT images. In the following section, the proposed algorithm will be introduced. In the third section, we will analyze the numerical simulation results and discuss relevant issues. In the last section, we will conclude the paper.

\section{Theory and method}

\subsection{CT reconstruction theory based on compressive sensing}

Theoretically, the mathematical CT model can be expressed as:

$$
A \vec{u}=\vec{p}
$$

where A is the system matrix, $\vec{u}$ is the original image, and $\vec{p}$ is the projection data. Traditional CT reconstruction algorithms such as FBP, ART and SART can't reconstruct high quality CT images with the sparse sampling or limited projection data.

In 2006, E.J. Candes and D.L. Donoho put forward the CS theory which makes it possible to get high quality CT images with sparse projection data [8]. The main idea of CS is that a signal can be reconstructed with far less sampled frequency than required by conventional Nyquist-Shannon sampling frequency, if the image has a sparse/compressible representation in a transform domain.

Compressive sensing theory can be expressed by the following equation:

$$
\min \|\vec{y}\|_{0} \text { s.t. } \quad \vec{p}=A \vec{u}=A \Phi^{H} \vec{y}
$$

where $\Phi$ is a orthogonal transform, $\Phi^{H}$ is the corresponding inverse transform, $\vec{u}$ is a CT image to be reconstructed and has a special relationship with $\Phi^{H}$, that is $\vec{u}=\Phi^{H} \vec{y} . \vec{p}$ is the projection data of $\vec{u}$ through matrix A.

\subsection{Total variation and adaptive-weighted diagonal total variation}

Inspired by CS theory, E.Y. Sidky and X.C. Pan proposed a TV-based on CT reconstruction algorithm using gradient operator as the sparse representation [10], in which TV is defined as follow: 


\begin{tabular}{|c|c|c|}
\hline$i-1, j-1$ & $i-1, j$ & $i-1, j+1$ \\
\hline$i, j-1$ & $i, j$ & $i, j+1$ \\
\hline$i+1, j-1$ & $i+1, j$ & $i+1, j+1$ \\
\hline
\end{tabular}

Fig. 1. The illustration of pixel position in an image.

$$
\|\vec{u}\|_{\mathrm{TV}}=\sum_{i, j} \sqrt{\left(u_{i, j}-u_{i-1, j}\right)^{2}+\left(u_{i, j}-u_{i, j-1}\right)^{2}}
$$

where $\|\vec{u}\|_{\mathrm{TV}}$ represents total variation of an image $\vec{u}, u_{i, j}$ is a pixel value of $\vec{u}, i$ and $j$ represent row and column respectively that is shown in Figure 1.

AwDTV is defined as follow:

$$
\|\vec{u}\|_{\text {AwDTV }}=\sum_{i, j} \sqrt{\omega_{i, i-1, j, j-1}\left(u_{i, j}-u_{i-1, j-1}\right)^{2}+\omega_{i, i-1, j, j+1}\left(u_{i, j}-u_{\mathrm{i}-1, j+1}\right)^{2}}
$$

where $\|\vec{u}\|_{\text {AwDTV }}$ represents adaptive-weighted diagonal total variation of an image $\vec{u}, \omega_{i, i-1, j, j-1}$ and $\omega_{i, i-1, j, j+1}$ are the adaptive weights which can be defined as:

$$
\omega_{i, i-1, j, j-1}=\exp \left[-\left(\frac{u_{i, j}-u_{i-1, j-1}}{\delta}\right)^{2}\right] \text { and } \omega_{i, i-1, j, j+1}=\exp \left[-\left(\frac{u_{i, j}-u_{\mathrm{i}-1, j+1}}{\delta}\right)^{2}\right]
$$

where $\delta$ is a scale factor which controls the strength of the diffusion during each iteration. In our study, we selected $\delta=0.21$.

\subsection{Proposed algorithm}

In this paper, we use AwDTV to represent images sparsely. We use the steepest descent method [18] to solve the optimization problem. The proposed algorithm can be defined as follow:

$$
\min \|\vec{u}\|_{\text {AwDTV }} \text { s.t. }\|A \vec{u}-\vec{p}\|_{2}^{2}<\sigma^{2}
$$

where $\sigma$ is permissible error of AwDTV.

The steepest descent method is applied to solve Eq. (6), we have the following formula:

$$
\vec{u}^{k+1}=\vec{u}^{k}-\alpha d \vec{g}_{\text {AwDTV }}^{k}
$$

where $k$ denotes the iteration index of AwDTV in the steepest descent method, $\alpha$ is gradient descent relaxing factor of AwDTV, $d$ is gradient descent scaling parameter of AwDTV. $\vec{u}^{0}$ is the initial 
image of AwDTV which is obtained by ART method, $\widehat{g}_{\text {AwDTV }}$ is the normalized AwDTV gradient while $\vec{g}_{\text {AwDTV }}$ is AwDTV gradient, and their relationship is $\hat{g}_{\text {AwDTV }}=\vec{g}_{\text {AwDTV }} /\left\|\vec{g}_{\text {AwDTV }}\right\|_{2}$. The individual elements of $\vec{g}_{\text {AwDTV }}$ can be got by taking the derivative of $\|\vec{u}\|_{\text {AwDTV }}$ with respect to each pixel value results in a singular expression. We considered $\omega$ which is referred to Eq. (4) as an adaptive weight, it can be pre-computed at current iteration for the AwDTV minimization at the next iteration. So the individual elements of $\vec{g}_{\text {AwDTV }}$ can be approximately expressed as:

$$
\begin{gathered}
\frac{\partial\|\vec{u}\|_{\mathrm{AwDTV}}}{\partial u_{i, j}} \approx \frac{\omega_{i, i-1, j, j-1}\left(u_{i, j}-u_{i-1, j-1}\right)+\omega_{i, i-1, j, j+1}\left(u_{i, j}-u_{i-1, j+1}\right)}{\sqrt{\varepsilon+\omega_{i, i-1, j, j-1}\left(u_{i, j}-u_{i-1, j-1}\right)^{2}+\omega_{i, i-1, j, j+1}\left(u_{i, j}-u_{i-1, j+1}\right)^{2}}} \\
-\frac{\omega_{i+1, i, j-1, j}\left(u_{i+1, j-1}-u_{i, j}\right)}{\sqrt{\varepsilon+\omega_{i+1, i, j-1, j}\left(u_{i+1, j-1}-u_{i, j}\right)^{2}+\omega_{i+1, i, j-1, j-2}\left(u_{i+1, j-1}-u_{i, j-2}\right)^{2}}} \\
-\frac{\omega_{i+1, i, j+1, j}\left(u_{\mathrm{i}+1, j+1}-u_{i, j}\right)}{\sqrt{\varepsilon+\omega_{i+1, i, j+1, j}\left(u_{\mathrm{i}+1, j+1}-u_{i, j}\right)^{2}+\omega_{i+1, i, j+1, j+2}\left(u_{\mathrm{i}+1, j+1}-u_{i, j+2}\right)^{2}}}
\end{gathered}
$$

where $\varepsilon$ is a known positive integer. In our study, we selected $\varepsilon=10^{-8}$.

The implementation steps of our proposed algorithm which is shown in Table 1 contain two loops, the outside loop operates ART and the inside loop operates AwDTV gradient descent. The flow chart is shown in Table 1 where $m=2, \ldots, M$ denotes the projection angles, $\vec{A}_{m}$ is $m$ th row vector and system matrix A includes $M$ row vectors $\vec{A}_{m}$. Accordingly, $M$ row vectors $p_{m}$ compose the projection-data vector $p, \lambda$ is convergence parameter of ART method. The outside loop is labeled by $n$ and $N_{i t e r}$ is the max number of reconstruction iteration. The inside loop is labeled by $k$ and $K$ is the iteration count for AwDTV minimization. In our study, we selected $K=10$, which can strike a good balance in the steepest descent, and generate good reconstruction results in the experiments.

\section{Numerical simulation}

In this section, we present our numerical simulation results. There are two sets of model: Shepp-Logan phantom and FORBILD head phantom $[19,20]$ are used to depict the performance of our algorithm. This paper uses the Root Mean Square Errors (RMSE) and Universal Quality Index (UQI) [21] to evaluate the quality of the reconstructed images. RMSE is defined as:

$$
\operatorname{RMSE}=\left(\frac{1}{M \times N} \sum_{0 \leq i<N} \sum_{0 \leq j<M}\left(u_{i, j}-u_{i, j}^{*}\right)^{2}\right)^{\frac{1}{2}}
$$

where $u_{i, j}$ and $u_{i, j}^{*}$ are the pixel values of original image $(\vec{u})$ and reconstructed image $\left(\vec{u}^{*}\right)$ at position $(i, j)$, respectively. The value of UQI is between -1 and 1 . When the reconstructed image is same as the original image, the value of UQI is 1 . UQI is defined as: 
Table 1

Implementation steps of AwDTV reconstruction

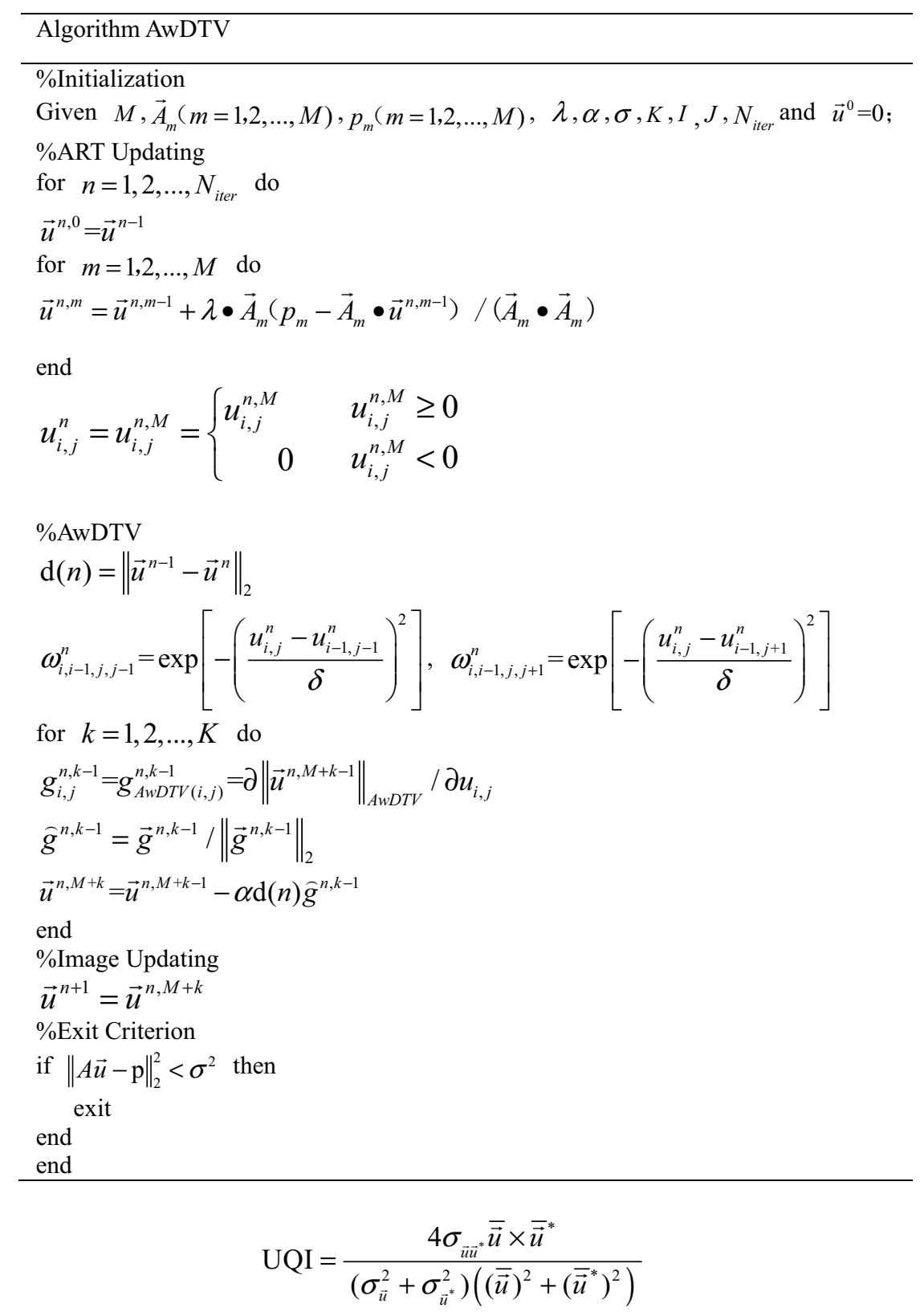

where $\overline{\vec{u}}$ and $\overline{\vec{u}}^{*}$ are the mean values of $\vec{u}$ and $\vec{u}^{*}$, respectively; $\sigma_{\vec{u}}^{2}$ and $\sigma_{\vec{u}}^{2}$ are variance of $\vec{u}$ and $\vec{u}^{*}$, respectively; $\sigma_{\vec{u} \vec{u}^{*}}$ is covariance of $\vec{u}$ and $\vec{u}^{*}$. 


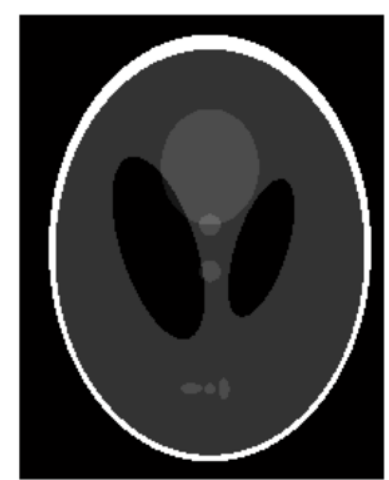

(a) Original Shepp-Logan phantom

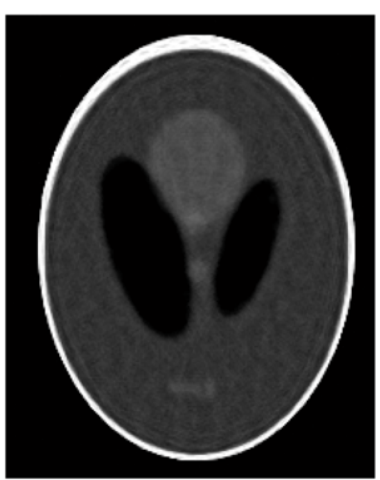

(b) ART

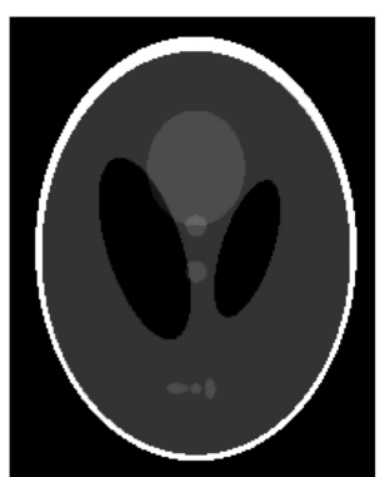

(c) TV

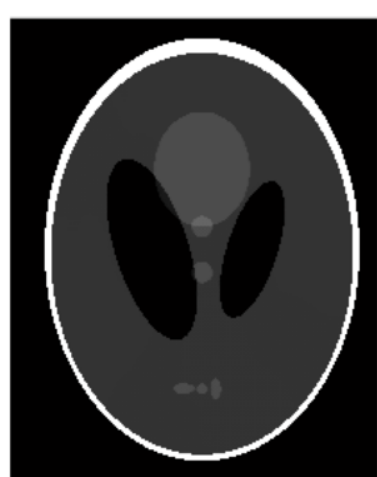

(d) AwDTV

Fig. 2. The reconstructed Shepp-Logan phantoms with three different algorithms from 20 projection views.

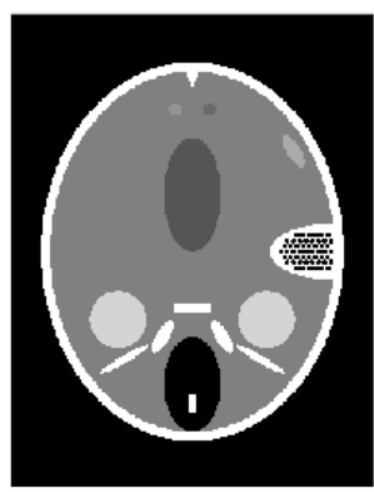

(a) original FORBILD head phantom

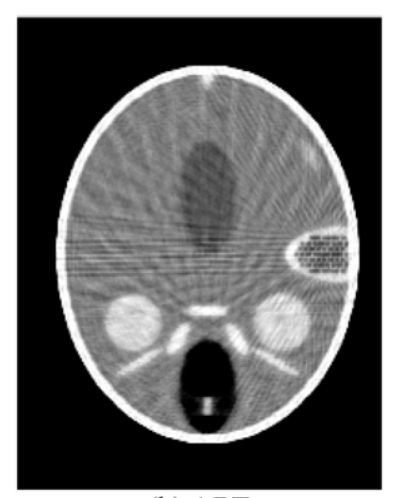

(b) ART

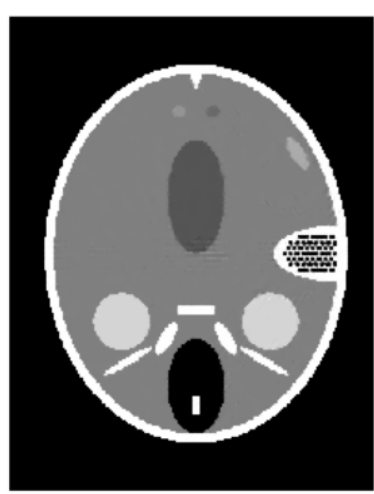

(c) TV

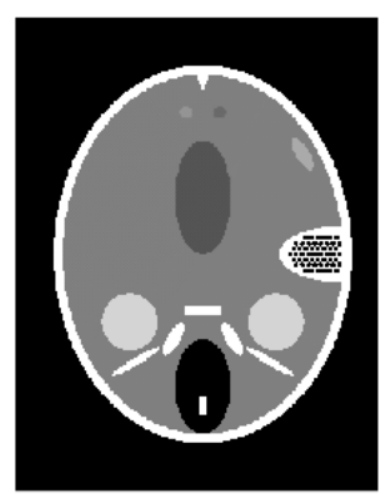

(d) AwDTV

Fig. 3. The reconstructed FORBILD head phantoms using three different reconstruction algorithms from 30 projection data.

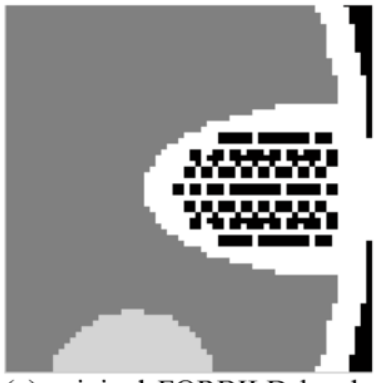

(a) original FORBILD head phantom

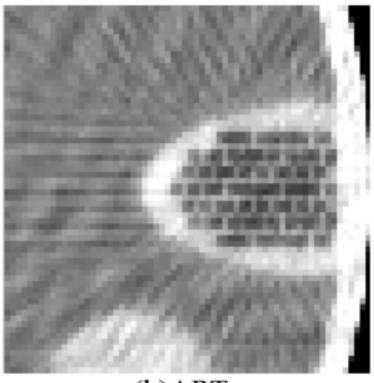

(b)ART

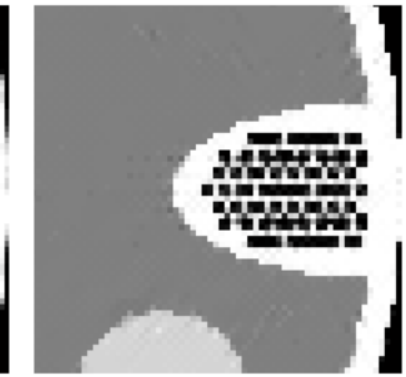

(c)TV

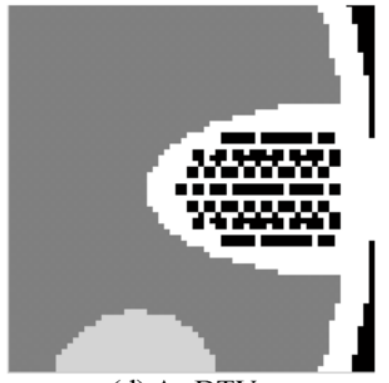

(d) AwDTV

Fig. 4. One magnified part of reconstructed FORBILD head phantoms using three different reconstruction algorithms from 30 projection data.

In the first simulation, a Shepp-Logan phantom as shown in Figure 2(a) is used to be reconstructed and compared with 3 different methods: ART, TV and AwDTV. The size of phantom image is $256 \times 256$. We assume that the CT system was viewed as in a typical pencil-beam geometry, and the 
scanning range was from 0 to $2 \pi$ with a $\theta$ angular increment. The projection number is 20 , which means $\theta=\pi / 10$. The iteration number is 1000 and all reconstructed images are shown in Figure 2.

From Figure 2, we can see that the reconstructed image using ART method contains a lot of artifacts. Though overall, TV and AwDTV have not much difference, the reconstructed image using AwDTV method contains less artifacts, and the inner distribution near edge is more uniform than the image reconstructed with TV.

In the second simulation, a FORBILD head phantom which has more details shown in Figure 3(a) is used to reconstruct and compared by 3 different methods: ART, TV and AwDTV, all reconstructed images are shown in Figure 3. ART method fails to reconstruct a high-quality image from 30 projection data and the reconstructed image contains a lot of artifacts as shown in Figure 3(b). TV method successfully suppresses the artifacts but introduces stair-case artifacts as shown in Figure 3(c). AwDTV method significantly suppresses the artifacts and preserves the edges as shown in Figure 3(d). To further observe, we zoom in one part of the reconstructed images as shown in Figure 4, and find the reconstructed image using AwDTV method contains less artifacts, and the inner distribution near edge is more uniform than the image reconstructed with TV. Figure 5 plots the RMSE and UQI with respect to iteration number, we can easily find that AwDTV always has lower RMSE and higher UQI with different iteration numbers.

Table 2 lists all the RMSE and UQI calculated from reconstructed Shepp-Logan phantom and FORBILD head phantom with 3 different approaches. It's obviously see that the RMSE of reconstructed images using AwDTV method is much smaller than that of reconstructed images using ART and TV methods, the UQI is much bigger. Thus AwDTV method can reconstruct images with higher quality.

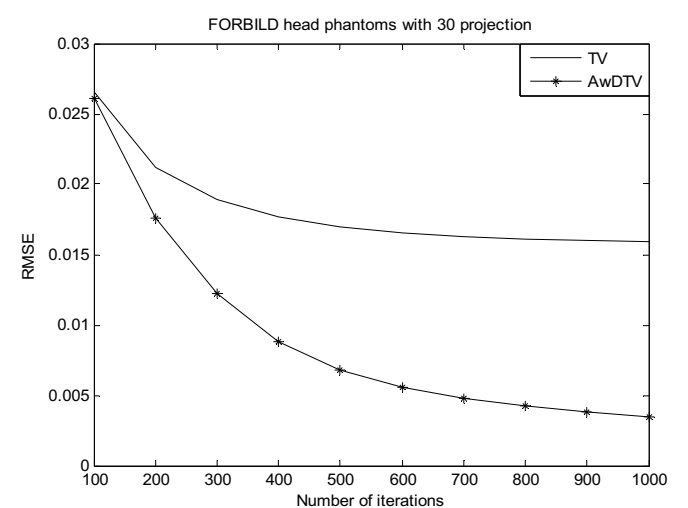

(a) RMSE

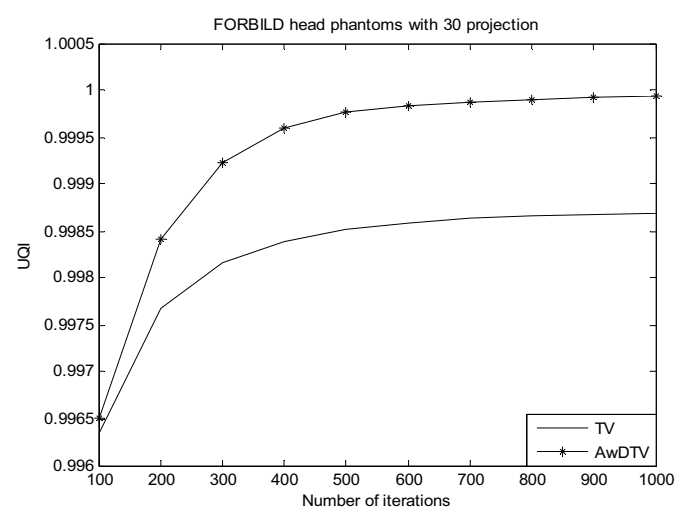

(b) UQI

Fig. 5. The RMSE and UQI lines of reconstructed FORBILD head phantoms with respect to iteration number (from 100 to 1000) for TV and AwDTV methods, where solid lines are the results of TV and asterisk lines are the results of AwDTV.

Table 2

Reconstruction results using Shepp-Logan phantom and FORBILD head phantom

\begin{tabular}{lllllll}
\hline & RMSE & \multicolumn{3}{c}{ UQI } \\
\hline Methods & ART & TV & AwDTV & ART & TV & AwDTV \\
& & & & & & \\
Shepp-Logan phantom & 0.0422 & 0.0066 & 0.0016 & 0.9800 & 0.9995 & 1.0000 \\
FORBILD head phantom & 0.0620 & 0.0159 & 0.0035 & 0.9799 & 0.9987 & 0.9999 \\
\hline
\end{tabular}




\section{Conclusion}

In this study, we proposed a CT reconstruction algorithm based on AwDTV. It uses the diagonal direction gradient to constraint reconstructed image and adds associated weights which are expressed as an exponential function and can be adaptively adjusted by the local image-intensity diagonal gradient for the purpose of preserving the edge details, then using the steepest descent method to solve the optimization problem. The numerical simulations demonstrate that the proposed method can reconstruct high-quality images from sparse-views data, AwDTV method has a potential in reducing the radiation dose in clinical application. In this study, we choose 1000 iterations which are also used by E.Y. Sidky and X.C. Pan who proposed TV method. The time of 1000 iterations depends on the specification of computer. The time consumption is 768 seconds on my computer. The TV method spends almost the same reconstruction time. It can be further improved with many methods such as parallel computing, CUDA acceleration, etc. In the further research, we will try to explore the directional problem of reconstructed image so as to find the best reconstructed direction of different images. Besides we will try to enhance the capability of anti-noise.

\section{Acknowledgment}

This work was supported in part by the National Natural Science Foundation of China (No. 61201346, No. 61401049 and No. 61471070), the Fundamental Research Funds for the Central Universities (No. CDJZR14125501), the Postdoctoral Science Foundation (No. 2014M560703), the Special Projects Funded for Postdoctoral Research of Chongqing City (No. Xm2014105) and Graduate Innovative Research Projects of Chongqing (CYB14024).

\section{References}

[1] G. Wang, H. Yu and M. De, An outlook on X-ray CT research and development, Medical Physics 35 (2008), 1051-1064.

[2] G. Li, S. Luo and N. Gu, Research progress of nano CT imaging, Chinese Science Bulletin 58 (2013), 501-509.

[3] H. Hiriyannaiah, X-ray computed tomography for medical imaging, IEEE Signal Processing Magazine 14 (1997), $42-59$.

[4] J. Chen, L. Liu, G. Liu, et al., High resolution X-ray microscopy and its new developments, Physics 36 (2007), 588-594.

[5] S. Zhang, W. Li and G. Tang, Study on image reconstruction algorithm of filtered backprojection, Journal of Xianyang Normal University 23 (2008), 47-49.

[6] R. Gordon, R. Bender and G. Herman, Algebraic reconstruction techniques (ART) for three-dimensional electron microscopy and X-ray photography, Journal of Theoretical Biology 29 (1970), 471-481.

[7] H. Andersen and A. Kak, Simultaneous algebraic reconstruction technique (SART): A superior implementation of the ART algorithm, Ultrasonic Imaging 6 (1984), 81-94.

[8] D. Donoho, Compressed sensing, IEEE Transactions on Information Theory 52 (2006), 1289-1306.

[9] E. Sidky, C. Kao and X. Pan, Accurate image reconstruction from few-views and limited-angle data in divergent-beam CT, Journal of X-Ray Science and Technology 14 (2006), 119-139.

[10] E.Y. Sidky and X.C. Pan, Image reconstruction in circular cone-beam computed tomography by constrained, total-variation minimization, Physics in Medicine and Biology 53 (2008), 4777-4807.

[11] Y. Liu, J. Ma, Y. Fan and Z. Liang, Adaptive-weighted total variation minimization for sparse data toward low-dose X-ray computed tomography image reconstruction, Physics in Medicine and Biology 57 (2012), 7923-7956.

[12] Y. Liu, Z. Liang, J. Ma, H. Lu, K. Wang, H. Zhang and W. Moore, Total variation-stokes strategy for sparse-view X-ray CT image reconstruction, IEEE Transactions on Medical Imaging 33 (2014), 749-763.

[13] M. Chen, D. Mi, P. He, L. Deng and B. Wei, A CT reconstruction algorithm based on L1/2 regularization, Computational and Mathematical Methods in Medicine 2014 (2014), 862910.

[14] M. Chang, L. Li, Z. Chen, Y. Xiao, L. Zhang and G. Wang, A few-view reweighted sparsity hunting (FRESH) method for 
CT image reconstruction, Journal of X-ray Science and Technology 21 (2013), 161-176.

[15] Q. Xu, H. Yu, X. Mou, L. Zhang, J. Hsieh and G. Wang, Low-dose X-ray CT reconstruction via dictionary learning, IEEE Transactions on Medical Imaging 31 (2012), 1682-1697.

[16] H. Li, X. Chen, Y. Wang, Z. Zhou, Q. Zhu and D. Yu, Sparse CT reconstruction based on multi-direction anisotropic total variation (MDATV), BioMedical Engineering OnLine 13 (2014), 1-27.

[17] L. Deng, P. Feng, M. Chen, P. He, Q. S. Vo and B. Wei, A CT reconstruction algorithm based on non-aliasing contourlet transform and compressive sensing, Computational and Mathematical Methods in Medicine 2014 (2014), 753615.

[18] H. Zhou and P. Wang, A simpler explicit iterative algorithm for a class of variational inequalities in hilbert spaces, Journal of Optimization Theory and Applications 161 (2014), 716-727.

[19] Z. Yu, F. Noo, F. Dennerlein, et al., Simulation tools for two dimensional experiments in X ray computed tomography using the FORBILD head phantom, Physics in Medicine and Biology 57 (2012), 237-252.

[20] http://www.imp.uni-erlangen.de/phantoms, last accessed June 1, 2015.

[21] Y. Liu, Research on objective full-reference image quality evaluation method, Engineering Master Dissertation, Nanjing University of Aeronautics and Astronautics, 2010. 